$\mathrm{UCLA} / 96 / \mathrm{TEP} / 24$

\title{
Understanding Chiral Anomaly in Coordinate Space *
}

\author{
Hidenori SONODA ${ }^{\dagger}$ \\ Department of Physics and Astronomy, UCLA, Los Angeles, CA 90095-1547, USA
}

\begin{abstract}
By completing the old discussion of $\mathrm{K}$. Wilson, we express the chiral anomaly in terms of a double integral of a three-point function of chiral currents over an arbitrarily small region in the coordinate space. An integrability condition provides an important finite local counterterm to the integral.
\end{abstract}

August 1996

* This work was supported in part by the U.S. Department of Energy, under Contract DEAT03-88ER 40384 Mod A006 Task C.

† E-mail: sonoda@physics.ucla.edu 
All known derivations of the chiral anomaly suggest the short-distance nature of the effect. In the derivation by Schwinger [1], the axial anomaly appears as an inevitable consequence of the short-distance singularity between two spinor fields and the vector gauge invariance. In another derivation [2] the anomaly arises from the ambiguity of a three-point function of chiral currents due to the linear divergence (in the momentum space) of a loop integral. If we regulate the linear divergence by the Pauli-Villars regulator, a local expression for the anomaly is obtained in the limit of the infinite regulator mass [3], which is equivalent to the zero distance limit.

The purpose of this little note is to complete and extend the old work of K. Wilson [4] in which the chiral anomaly was analyzed in the coordinate space. Though the anomaly was not evaluated explicitly in ref. [4], the origin of the anomaly was correctly identified as the subtlety in defining a double integral of a three-point function of chiral currents caused by short distance singularities. The final expression we will obtain for the anomaly is valid not only for the free theory but also for the interacting theories like QCD. To facilitate the derivation we will use the euclidean space instead of the Minkowski space.11

We first review consistent and covariant chiral currents. For definiteness we consider QCD with $N_{f}$ massless quarks. If we couple a classical SU $\left(N_{f}\right)$ gauge field $A$ to the righthanded currents, the vacuum energy $W[A]$ becomes a functional of the external gauge field. The consistent current is defined so that its expectation value gives the first order variation of the vacuum energy:

$$
W[A+\delta A]-W[A]=\int d^{4} r \delta A_{\mu}^{A}(r)\left\langle J_{\mu}^{A}(r)\right\rangle_{A}
$$

The consistent current $J_{\mu}^{A}$ satisfies the anomaly equation:

$$
D_{\mu} J_{\mu}^{A} \equiv \partial_{\mu} J_{\mu}^{A}+f^{A B C} A_{\mu}^{B} J_{\mu}^{C}=c \epsilon_{\mu \nu \alpha \beta} \partial_{\mu} \operatorname{tr}\left(-i T^{A}\right)\left(A_{\nu} \partial_{\alpha} A_{\beta}+\frac{1}{2} A_{\nu} A_{\alpha} A_{\beta}\right)
$$

where $c$ is a constant, $A_{\mu} \equiv-i T^{A} A_{\mu}^{A},\left[T^{A}, T^{B}\right]=i f^{A B C} T^{C}$, and the hermitian generators are normalized as $\operatorname{tr} T^{A} T^{B}=\delta^{A B}$. The consistent current $J_{\mu}^{A}$ does not transform covariantly under the flavor gauge transformation. To make it covariant, we must add a counterterm:

$$
\tilde{J}_{\mu}^{A} \equiv J_{\mu}^{A}+\Delta J_{\mu}^{A}
$$

1 The chiral anomaly has been discussed and computed in the framework of differential renormalization in coordinate space [5]. Our approach is more directly related to ref. [4]. 
where

$$
\Delta J_{\mu}^{A} \equiv c \epsilon_{\mu \nu \alpha \beta} \operatorname{tr}\left(-i T^{A}\right)\left(\partial_{\nu} A_{\alpha} A_{\beta}+A_{\nu} \partial_{\alpha} A_{\beta}+\frac{3}{2} A_{\nu} A_{\alpha} A_{\beta}\right)
$$

The covariant current then satisfies

$$
D_{\mu} \tilde{J}_{\mu}^{A}=\frac{3 c}{4} \operatorname{tr}\left(-i T^{A}\right) F_{\mu \nu} \tilde{F}_{\mu \nu}
$$

where $F_{\mu \nu}$ is the field strength, and $\tilde{F}_{\mu \nu} \equiv \epsilon_{\mu \nu \alpha \beta} F_{\alpha \beta}$.

Our goal is to compute the covariant divergence of the covariant current $D_{\mu}\left\langle\tilde{J}_{\mu}^{A}\right\rangle_{A}$ to second order in the external gauge field. In order to calculate this in the coordinate space, we need a formula that expresses the first order variation of the n-point function of the covariant currents. The formula is given by

$$
\begin{aligned}
& \left\langle\tilde{J}_{\mu_{1}}^{A_{1}}\left(r_{1}\right) \ldots \tilde{J}_{\mu_{n}}^{A_{n}}\left(r_{n}\right)\right\rangle_{A+\delta A}-\left\langle\tilde{J}_{\mu_{1}}^{A_{1}}\left(r_{1}\right) \ldots \tilde{J}_{\mu_{n}}^{A_{n}}\left(r_{n}\right)\right\rangle_{A} \\
& =-\int_{\left|r-r_{k}\right| \geq \epsilon_{k}} d^{4} r \delta A_{\mu}^{A}(r)\left\langle\left(\tilde{J}_{\mu}^{A}(r)-\left\langle\tilde{J}_{\mu}^{A}(r)\right\rangle_{A}\right) \tilde{J}_{\mu_{1}}^{A_{1}}\left(r_{1}\right) \ldots \tilde{J}_{\mu_{n}}^{A_{n}}\left(r_{n}\right)\right\rangle_{A} \\
& \quad-\sum_{k=1}^{n}\left[\delta A \cdot \mathcal{S}\left(\epsilon_{k} ; A\left(r_{k}\right)\right)\right]_{\mu_{k}}^{A_{k}}\left\langle\tilde{J}_{\mu_{1}}^{A_{1}}\left(r_{1}\right) \ldots \tilde{J}_{\mu_{k}} \widehat{A_{k}}\left(r_{k}\right) \ldots \tilde{J}_{\mu_{n}}^{A_{n}}\left(r_{n}\right)\right\rangle_{A} .
\end{aligned}
$$

The limit $\epsilon_{k} \rightarrow 0$ can be taken independently as long as the points $r_{k}$ are all different. The c-number counterterm $\delta A \cdot \mathcal{S}$ is necessary to subtract the short-distance singularities contained in the product of two chiral currents. The possible form of the counterterm is restricted by covariance, dimensionality, and $\mathrm{CP}$ invariance. The most general form is given by

$$
\begin{aligned}
& {[\delta A \cdot \mathcal{S}(\epsilon ; A)]_{\mu}^{A}} \\
& =\operatorname{tr}\left(-i T^{A}\right)\left[c_{1}(\epsilon) \delta A_{\mu}+c_{2}(\epsilon)\left[\delta A_{\nu}, F_{\mu \nu}\right]+c_{3}(\epsilon) \frac{1}{2} D_{\alpha} D_{\alpha} \delta A_{\mu}\right. \\
& \left.\quad+c_{4}(\epsilon) \frac{1}{2}\left(D_{\mu} D_{\nu}+D_{\nu} D_{\mu}\right) \delta A_{\nu}+\tilde{c}(\epsilon)\left\{\delta A_{\nu}, \tilde{F}_{\mu \nu}\right\}\right],
\end{aligned}
$$

where we denote the $\epsilon$ dependence of the counterterms explicitly. For the derivation of the anomaly, only the term proportional to $\tilde{c}(\epsilon)$ will play a role.

Before we apply the variational formula (6) to the chiral anomaly, we determine the counterterm $\tilde{c}(\epsilon)$. The second order variation of the vacuum energy $W\left[A+\delta_{1} A+\delta_{2} A\right]-$ $W\left[A+\delta_{1} A\right]-W\left[A+\delta_{2} A\right]+W[A]$ can be calculated by using eqn. (1) with respect to $\delta_{1} A$, and then by using the variational formula (6) with respect to $\delta_{2} A$. The integrability 
of the vacuum energy demands that the second order variation be symmetric with respect to $\delta_{1} A$ and $\delta_{2} A$. This gives

$$
\begin{aligned}
\int & d^{4} r\left(\delta_{2} A_{\mu}^{A}(r)\left[\delta_{1} A \cdot \mathcal{S}(\epsilon ; A(r))\right]_{\mu}^{A}-(1 \leftrightarrow 2)\right) \\
& =\int d^{4} r\left(\delta_{1} A_{\mu}^{A}(r)\left(\Delta J_{\mu}^{A}\left(r ; A+\delta_{2} A\right)-\Delta J_{\mu}^{A}(r ; A)\right)-(1 \leftrightarrow 2)\right) .
\end{aligned}
$$

By calculating the right-hand side using the explicit form (4), we obtain

$$
\begin{aligned}
\delta_{2} A_{\mu}^{A}\left[\delta_{1} A \cdot \mathcal{S}(\epsilon ; A)\right]_{\mu}^{A}-(1 \leftrightarrow 2) \\
\quad=\frac{3 c}{2} \epsilon_{\mu \nu \alpha \beta} \operatorname{tr}\left(\delta_{1} A_{\mu} \delta_{2} A_{\nu}-\delta_{2} A_{\mu} \delta_{1} A_{\nu}\right) F_{\alpha \beta}+\text { (total derivatives) } .
\end{aligned}
$$

Substituting eqn. (7) into the left-hand side, we obtain

$$
\tilde{c}(\epsilon)=-\frac{3 c}{4}
$$

a constant independent of $\epsilon$.

Now we are ready to derive an expression for the chiral anomaly. By applying the variational formula (6) twice, we can compute $D_{\mu}\left\langle\tilde{J}_{\mu}^{A}\right\rangle_{A}$ up to second order in the external gauge field:

$$
\begin{aligned}
& D_{\mu}\left\langle\tilde{J}_{\mu}^{A}(0)\right\rangle_{A} \simeq \\
& -\frac{1}{2} \int d^{3} \Omega_{\epsilon}(r) A_{\nu}^{B}(r) \hat{r}_{\mu} \int_{\substack{r^{\prime} \geq \eta \\
\left|r^{\prime}-r\right| \geq \kappa}} d^{4} r^{\prime} A_{\alpha}^{C}\left(r^{\prime}\right)\left\langle J_{\alpha}^{C}\left(r^{\prime}\right) J_{\nu}^{B}(r) J_{\mu}^{A}(0)\right\rangle_{A=0}^{c} \\
& \quad-\frac{1}{2}\left(c_{2}(\epsilon)+\frac{c_{3}(\epsilon)+c_{4}(\epsilon)}{2}\right) \operatorname{tr}\left(-i T^{A}\right)\left[A_{\mu}, \partial^{2} A_{\mu}\right] \\
& \quad-\frac{1}{2}\left(-c_{2}(\epsilon)+\frac{c_{3}(\epsilon)+3 c_{4}(\epsilon)}{2}\right) \operatorname{tr}\left(-i T^{A}\right)\left[A_{\mu}, \partial_{\mu} \partial_{\nu} A_{\nu}\right] \\
& \quad+\frac{3 c}{4} \epsilon_{\mu \nu \alpha \beta} \operatorname{tr}\left(-i T^{A}\right)\left\{\partial_{\mu} A_{\nu}, \partial_{\alpha} A_{\beta}\right\} \\
& -\frac{1}{2}\left(f^{A B D} A_{\mu}^{B}(0) \int_{r^{\prime} \geq \eta} d^{4} r^{\prime} A_{\alpha}^{C}\left(r^{\prime}\right)\left\langle J_{\alpha}^{C}\left(r^{\prime}\right) J_{\mu}^{D}(0)\right\rangle_{A=0}^{c}\right. \\
& \left.+\operatorname{tr}\left(-i T^{A}\right)\left[A_{\mu}, \frac{c_{3}(\eta)}{2} \partial^{2} A_{\mu}+c_{4}(\eta) \partial_{\mu} \partial_{\nu} A_{\nu}\right]\right)
\end{aligned}
$$

where $\hat{r}_{\mu} \equiv r_{\mu} / r, d^{3} \Omega_{\epsilon}(r)$ is the solid angle element at radius $r=\epsilon$, and the limits $\kappa \rightarrow 0$ and $\eta \rightarrow 0$ must be taken before the limit $\epsilon \rightarrow 0$. 
In deriving the above formula (11), we used the relation

$$
\begin{aligned}
\frac{\partial}{\partial r_{\mu}} & \int_{\left|r^{\prime}-r\right| \geq \epsilon} d^{4} r^{\prime} A_{\nu}^{B}\left(r^{\prime}\right)\left\langle J_{\nu}^{B}\left(r^{\prime}\right) J_{\mu}^{A}(r)\right\rangle \\
= & \int_{\left|r^{\prime}-r\right| \geq \epsilon} d^{4} r^{\prime} A_{\nu}^{B}\left(r^{\prime}\right)\left\langle J_{\nu}^{B}\left(r^{\prime}\right) \partial_{\mu} J_{\mu}^{A}(r)\right\rangle \\
& -\int d^{3} \Omega_{\epsilon}\left(r^{\prime}-r\right) A_{\nu}^{B}\left(r^{\prime}\right) \frac{\left(r^{\prime}-r\right)_{\mu}}{\epsilon}\left\langle J_{\nu}^{B}\left(r^{\prime}\right) J_{\mu}^{A}(r)\right\rangle,
\end{aligned}
$$

which is the euclidean analogue of the well-known formula in Minkowski space for the time derivative of the time-ordered product:

$$
\partial_{\mu} \mathbf{T} J^{\mu}(t, \vec{x}) J^{\nu}(0)=\mathbf{T} \partial_{\mu} J^{\mu}(t, \vec{x}) J^{\nu}(0)+\delta(t)\left[J^{0}(0, \vec{x}), J^{\nu}(0)\right]
$$

We can simplify the second order expression (11) in two steps. First using the OPE

$$
\begin{aligned}
& \lim _{\epsilon \rightarrow 0} \int d^{3} \Omega_{\epsilon}(r) A_{\nu}^{B}(r) \hat{r}_{\mu}\left\langle J_{\alpha}^{C}\left(r^{\prime}\right) J_{\nu}^{B}(r) J_{\mu}^{A}(0)\right\rangle_{A=0}^{c} \\
& \quad=-f^{A B D} A_{\mu}^{B}(0)\left\langle J_{\alpha}^{C}\left(r^{\prime}\right) J_{\mu}^{D}(0)\right\rangle_{A=0}^{c},
\end{aligned}
$$

which is the euclidean analogue of the current-current commutation relation, we can restrict the range of integration over $r^{\prime}$ to $r_{0} \geq r^{\prime}$, where $r_{0}$ is an arbitrary finite radius. This assures the locality of the anomaly, since $r_{0}$ can be as small as we want. Second, we can take the gauge potential at $r=0$ as vanishing:

$$
A(r=0)=0 .
$$

This is because those contributions from the double integral which are proportional to $A(0)$ are canceled by the counterterms. We can understand this cancelation as a consequence of the covariance under the external gauge transformations. After these simplifications, we obtain the main result of this note:

$$
\begin{aligned}
D_{\mu} & \left\langle\tilde{J}_{\mu}^{A}(0)\right\rangle_{A} \\
\simeq & -\frac{1}{2} \partial_{\sigma} A_{\nu}^{B} \partial_{\tau} A_{\alpha}^{C} \lim _{\epsilon \rightarrow 0} \int d^{3} \Omega_{\epsilon}(r) r_{\sigma} \hat{r}_{\mu} \\
& \times \lim _{\eta, \kappa \rightarrow 0} \int_{\substack{r_{0} \geq r^{\prime} \geq \eta \\
\left|r^{\prime}-r\right| \geq \kappa}} d^{4} r^{\prime} r_{\tau}^{\prime}\left\langle J_{\alpha}^{C}\left(r^{\prime}\right) J_{\nu}^{B}(r) J_{\mu}^{A}(0)\right\rangle_{A=0}^{c} \\
& +\frac{3 c}{4} \epsilon_{\mu \nu \alpha \beta} \operatorname{tr}\left(-i T^{A}\right)\left\{\partial_{\mu} A_{\nu}, \partial_{\alpha} A_{\beta}\right\} .
\end{aligned}
$$


It is important to note the contribution of the counterterm. The radius $r_{0}$ is arbitrary, and the locality of the anomaly is manifest. The above expression for the anomaly is valid not only for the free theory but also for the interacting theories like QCD with quarks. We can appreciate the subtlety of the double limit by noticing, as in [4], that if we take the limit $\epsilon \rightarrow 0$ before $\eta \rightarrow 0$, the entire double integral in eqn. (11) is canceled by the single integral due to the OPE (14).

By combining eqn. (16) with the expected covariant anomaly equation (5), we can obtain the following formula that determines the coefficient $c$ in terms of a double integral 2 :

$$
\begin{aligned}
& \partial_{\sigma} A_{\nu}^{B} \partial_{\tau} A_{\alpha}^{C} \lim _{\epsilon \rightarrow 0} \int d^{3} \Omega_{\epsilon}(r) r_{\sigma} \hat{r}_{\mu} \\
& \quad \times \lim _{\eta, \kappa \rightarrow 0} \int_{\substack{r_{0} \geq r^{\prime} \geq \eta \\
\left|r^{\prime}-r\right| \geq \kappa}} d^{4} r^{\prime} r_{\tau}^{\prime}\left\langle J_{\alpha}^{C}\left(r^{\prime}\right) J_{\nu}^{B}(r) J_{\mu}^{A}(0)\right\rangle_{A=0}^{c} \\
& =-\frac{3 c}{2} \epsilon_{\sigma \nu \tau \alpha} \operatorname{tr}\left(-i T^{A}\right)\left\{\partial_{\sigma} A_{\nu}, \partial_{\tau} A_{\alpha}\right\} .
\end{aligned}
$$

Without the contribution of the counterterm in eqn. (16), we would get a wrong factor $-3 c$ on the right-hand side.

Since QCD is asymptotic free, we can evaluate the coefficient $c$ using the free fermion theory. For the free right-handed currents, we obtain

$$
\left\langle J_{\alpha}^{C}\left(r^{\prime}\right) J_{\nu}^{B}(r) J_{\mu}^{A}(0)\right\rangle_{f r e e}^{c}=\frac{\left(r^{\prime}-r\right)_{\beta} r_{\gamma} r_{\delta}^{\prime}}{r^{\prime 4} r^{4}\left(r^{\prime}-r\right)^{4}} T_{\alpha \nu \mu, \beta \gamma \delta}^{C B A}
$$

where

$$
T_{\alpha \nu \mu, \beta \gamma \delta}^{C B A} \equiv \frac{i}{8 \pi^{6}}\left(\operatorname{tr} T^{C} T^{B} T^{A} \operatorname{Sp} \frac{1-\gamma_{5}}{2} \gamma_{\alpha} \gamma_{\beta} \gamma_{\nu} \gamma_{\gamma} \gamma_{\mu} \gamma_{\delta}-(B \leftrightarrow C, \alpha \leftrightarrow \nu, \gamma \leftrightarrow \delta)\right)
$$

Using the formula

$$
\begin{aligned}
& \lim _{\eta, \kappa \rightarrow 0} \int_{\substack{r_{0} \geq r^{\prime} \geq \eta \\
\left|r^{\prime}-r\right| \geq \kappa}} d^{4} r^{\prime} \frac{\left(r^{\prime}-r\right)_{\beta} r_{\delta}^{\prime} r_{\tau}^{\prime}}{r^{\prime 4}\left(r^{\prime}-r\right)^{4}} \\
& \quad=\frac{\pi^{2}}{r}\left[\left(\frac{1}{4}-\frac{r^{2}}{6 r_{0}^{2}}\right)\left(\delta_{\tau \beta} \hat{r}_{\delta}+\delta_{\delta \beta} \hat{r}_{\tau}\right)+\left(-\frac{1}{4}+\frac{r^{2}}{12 r_{0}^{2}}\right) \delta_{\tau \delta} \hat{r}_{\beta}-\frac{1}{2} \hat{r}_{\beta} \hat{r}_{\tau} \hat{r}_{\delta}\right]
\end{aligned}
$$

it is straightforward to find the well-known answer

$$
c=\frac{1}{24 \pi^{2}} \text {. }
$$

In lieu of conclusions, the author wishes to mention that the present work is a byproduct of his recent efforts [6] to reformulate quantum field theory in coordinate space with the help of the first order variational formulas analogous to eqn. (6).

2 This formula gives the euclidean analogue of the formulas $(7.19-21)$ of ref. 4 . 


\section{References}

[1] J. Schwinger, Phys. Rev. 82(1951)664

[2] S. L. Adler, Phys. Rev. 177(1969)2426;

J. Bell and R. Jackiw, Nuovo Cimento 60A(1969)47;

W. Bardeen, Phys. Rev. 184(1969)1848

[3] R. Jackiw, in "Lectures on Current Algebra and Its Applications," Princeton University Press (Princeton, NJ 1972)

[4] K. G. Wilson, Phys. Rev. 179(1969)1499

[5] D. Z. Freedman, K. Johnson, and J. I. Lattore, Nucl. Phys. B371(1992)353

[6] H. Sonoda, "The Energy-Momentum Tensor in Field Theory I, II," hep-th/9504113, 9509018 